\title{
An example for the holomorphic sectional curvature of the Bergman metric
}

\author{
by ŻYwOMIR Dinew (Kraków)
}

\begin{abstract}
We study the behaviour of the holomorphic sectional curvature (or Gaussian curvature) of the Bergman metric of planar annuli. The results are then utilized to construct a domain for which the curvature is divergent at one of its boundary points and moreover the upper limit of the curvature at that point is maximal possible, equal to 2 , whereas the lower limit is $-\infty$.
\end{abstract}

0. Introduction. Recall that the holomorphic sectional curvature of the Bergman metric of a bounded pseudoconvex domain $U \subset \mathbb{C}^{n}$ at the point $z \in U$ in direction $X \in \mathbb{C}^{n}$ is defined as follows:

$$
R_{U}(z, X):=\left(\sum_{p, q=1}^{n} g_{p \bar{q}} X_{p} \overline{X_{q}}\right)^{-2} \sum_{i, j, k, l=1}^{n} R_{\bar{i} j k \bar{l}} \overline{X_{i}} X_{j} X_{k} \overline{X_{l}}
$$

here

$$
R_{\overline{i j k l}}:=-\frac{\partial^{2} g_{\bar{j} \bar{i}}}{\partial z_{k} \partial \bar{z}_{l}}+\sum_{r, s=1}^{n} g^{\bar{r} s} \frac{\partial g_{j \bar{r}}}{\partial z_{k}} \frac{\partial g_{s \bar{i}}}{\partial \bar{z}_{l}}
$$

where $g^{\bar{r} s}$ stands for the $(r, s)$ th entry of the inverse matrix of $g_{p \bar{q}}$. The term in brackets in the definition of $R_{U}$ is introduced for the sake of normalization. Finally $g_{p \bar{q}}$ stands for $\frac{\partial^{2}}{\partial z_{p} \partial \bar{z}_{q}} \log \mathrm{K}_{U}(z, z)$, where $\mathrm{K}_{U}(z, z)$ is the Bergman kernel (on the diagonal) of the domain $U$.

One can show that

$$
R_{U}\left(z_{0}, X\right)=2-\frac{J_{0, U}\left(z_{0} ; X\right) J_{2, U}\left(z_{0} ; X\right)}{J_{1, U}\left(z_{0} ; X\right)^{2}},
$$

2010 Mathematics Subject Classification: Primary 32A36, 32W05; Secondary 32F45, 30A31, 30C40.

Key words and phrases: holomorphic sectional curvature, Bergman metric. 
where

$$
\begin{gathered}
J_{0, U}\left(z_{0} ; X\right):=\sup \left\{\left|f\left(z_{0}\right)\right|^{2}: f \in \mathcal{O}(U) \cap L^{2}(U), \int_{U}|f|^{2} \leq 1\right\}, \\
J_{1, U}\left(z_{0} ; X\right):=\sup \left\{\left|\sum_{j=1}^{n} \frac{\partial f\left(z_{0}\right)}{\partial z_{j}} X_{j}\right|^{2}: f \in \mathcal{O}(U) \cap L^{2}(U),\right. \\
J_{2, U}\left(z_{0} ; X\right):=\sup \left\{\left|\sum_{U}^{n} \frac{\partial^{2} f\left(z_{0}\right)}{\partial z_{j} \partial z_{i}} X_{j} X_{i}\right|^{2}: f \in \mathcal{O}(U) \cap L^{2}(U),\right. \\
\left.\int_{U}|f|^{2} \leq 1, f\left(z_{0}\right)=0\right\},
\end{gathered}
$$

We see that $J_{0, U}\left(z_{0} ; X\right)=\mathrm{K}_{U}\left(z_{0}, z_{0}\right)$, which is independent of $X$, and that the holomorphic sectional curvature of the Bergman metric is invariant under biholomorphic mappings.

From 0.2 it follows immediately that

$$
R_{U}(z, X)<2, \quad z \in U .
$$

This was already established by Bergman. It was shown by Lebed' (see [12]) that when $n \geq 2$ this estimate is optimal in the following weak sense: For each $\varepsilon>0$ there exists a domain $U_{\varepsilon}$ for which there exist $z \in U_{\varepsilon}$ and $X \in \mathbb{C}^{n}$ such that $R_{U_{\varepsilon}}(z, X)>2-\varepsilon$. In a very recent paper Chen and Lee ([5]) have shown that the estimate is optimal in the strong sense, i.e., there exists a domain $U$ and $z_{0} \in \partial U$ such that $\lim _{\nu \rightarrow \infty} R_{U}\left(z_{\nu}, X\left(z_{\nu}\right)\right)=2$ for suitably chosen $z_{\nu} \in U$ with $z_{\nu} \rightarrow z_{0}$ and $X\left(z_{\nu}\right)$. The question of the existence of a lower bound is also answered (in the negative) in higher dimensions in the paper of Herbort (see [9], where even an example with smooth boundary is provided).

In dimension 1 the formula 0.1 becomes

$$
R_{U}(z, X)=\frac{-g_{\overline{1} 11 \overline{1}}+\frac{g_{\overline{1} 1 \overline{1}} g_{1 \overline{1} 1}}{g_{1 \overline{1}}}}{\left(g_{1 \overline{1}}\right)^{2}}=\frac{-\left(\log g_{1 \overline{1}}\right)_{1 \overline{1}}}{g_{1 \overline{1}}}
$$

which is independent of $X$, and therefore we will use the shorter form $R_{U}(z)$. In fact this is exactly the Gaussian curvature of $g$.

Little is known about the holomorphic sectional curvature of the Bergman metric in dimension one. This is mainly because for most of the planar domains one cannot compute the Bergman kernel explicitly. The first nontrivial (i.e., not biholomorphic to the unit disc) domain for which one can say more is the circular annulus. 
The Bergman kernel of the annulus $P_{r}=\{z \in \mathbb{C}: r<|z|<1\}$ is

$$
\mathrm{K}_{P_{r}}(z, z)=-\frac{1}{\pi|z|^{2} \log \left(r^{2}\right)}+\pi^{-1} \sum_{j=0}^{\infty}\left(\frac{r^{2+2 j}}{\left(-r^{2+2 j}+|z|^{2}\right)^{2}}+\frac{r^{2 j}}{\left(1-r^{2 j}|z|^{2}\right)^{2}}\right)
$$

(see e.g. [10]). This will be denoted as $\mathrm{K}$ for short.

Because the annulus has smooth boundary, it follows by a theorem of Klembeck (see [11]) that

$$
\lim _{P_{r} \ni z \rightarrow \partial P_{r}} R_{P_{r}}(z)=-1 .
$$

When $r \rightarrow 0$, the domains $P_{r}$ will exhaust the punctured unit disc and therefore one can expect the corresponding holomorphic sectional curvatures of the Bergman metric to be convergent to the curvature of the punctured disc which is the same as for the whole disc (the constant -1). (In the case of the Bergman kernel this is Ramadanov's theorem.) This is indeed the case, but the convergence is only locally uniform. Moreover, numerical experiments of the author have shown that when $r$ gets smaller, the global maximum of the holomorphic sectional curvature of the Bergman metric becomes closer to 2 , and the global minimum tends to be unbounded.

The figures below present the behaviour of the curvature, when restricted to the line segment $(r, 1) \subset \mathbb{R}$, for different choices of $r$. (Figures 3 and 4 are for the same $r=0.001$, but Figure 4 is scaled in order to focus on the global maximum).

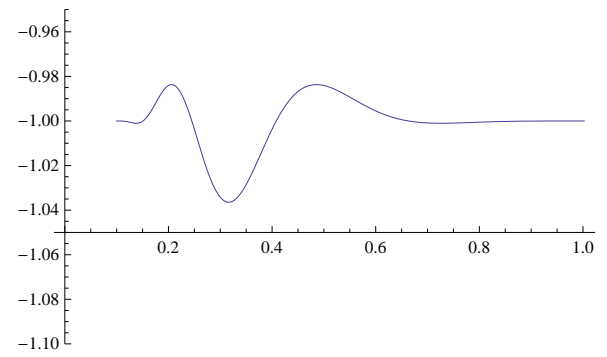

Fig. 1. The curvature of $P_{0.1}$ restricted to $(0.1,1)$

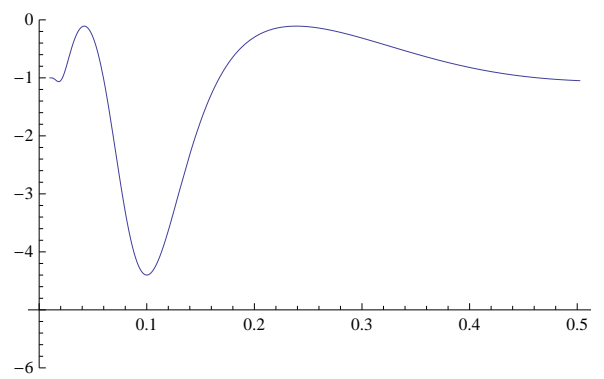

Fig. 2. The curvature of $P_{0.01}$ restricted to $(0.01,1)$ 


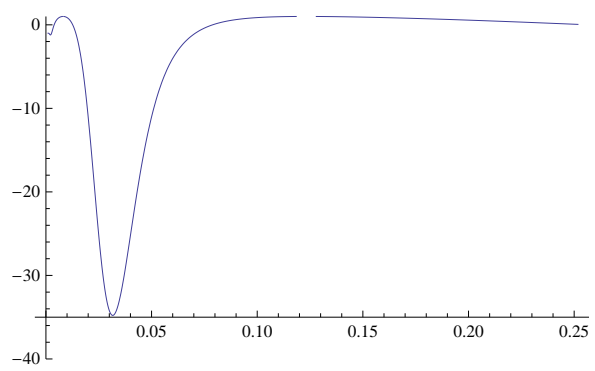

Fig. 3. The curvature of $P_{0.001}$ restricted to $(0.001,1)$

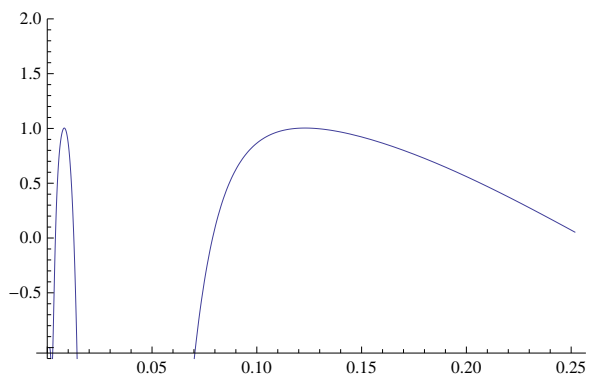

Fig. 4. The curvature of $P_{0.001}$ restricted to $(0.001,1)$

We will confirm these numerical experiments by analytically proving the following result:

TheOREM 0.1. For the circular annuli $P_{r}$ one has

$$
\lim _{r \rightarrow 0} R_{P_{r}}(\sqrt{r})=-\infty, \quad \lim _{r \rightarrow 0} R_{P_{r}}\left(r^{3 / 10}\right)=2 .
$$

Section 1 is entirely devoted to the very technical but rigorous proof of this theorem $\left({ }^{1}\right)$.

This shows that Lebed's result can be extended to $n=1$ and that one cannot find a universal constant that would be a lower bound for any planar domain.

In Section 2 the result of Section 1 is utilized to construct a planar domain for which the holomorphic sectional curvature of the Bergman metric is divergent at one of the boundary points of the domain. Namely one has

THEOREM 0.2. There exists a bounded planar domain $\Omega$ and a point $\zeta \in \partial \Omega$ such that

$$
\limsup _{\Omega \ni z \rightarrow \zeta} R_{\Omega}(z)=2 \quad \text { and } \quad \liminf _{\Omega \ni z \rightarrow \zeta} R_{\Omega}(z)=-\infty .
$$

$\left({ }^{1}\right)$ W. Zwonek obtained a considerably easier proof (see [15]). He moreover obtained a full characterization of the boundary behaviour of $R_{P_{r}}$, for all exponents of $r$ (not only $1 / 2$ and $3 / 10$ as above). 
This is done by using the known localization technique (in the style of [4] or 8]) which heavily depends on the geometry of the domain.

This proves that the constant 2 is optimal in the strong sense even in dimension 1 and that planar domains with unbounded holomorphic sectional curvature of the Bergman metric do exist. Here of course the domain cannot have smooth boundary (because of the aforementioned theorem of Klembeck), nor can it be finitely connected (because of the uniformization theorem which essentially reduces the problem to the first case). On the other hand the theory of Bergman invariants on nonsmooth domains is far less clear, compared to the smooth case.

In the whole paper $G_{\Omega}\left(z, z_{0}\right)$ will stand for the (negative) Green function i.e.,

$G_{\Omega}\left(z, z_{0}\right):=\sup \left\{u(z): u \in S H(\Omega), u \leq 0, \lim _{w \rightarrow z_{0}}\left(u(w)-\log \left|w-z_{0}\right|\right)<\infty\right\}$,

where $S H$ stands for subharmonic. Note that it is more common to call $-G\left(z, z_{0}\right)$ "Green function", but we will stick to the above definition.

\section{Behaviour of the curvature of circular annuli. Let}

$$
S:=-\frac{\mathrm{K}_{\overline{1}} \mathrm{~K}_{1}}{\mathrm{~K}^{2}}+\frac{\mathrm{K}_{1 \overline{1}}}{\mathrm{~K}} .
$$

Using (0.7) we expand explicitly $R_{P_{r}}$ by means of consecutive derivatives of the Bergman kernel $\mathrm{K}$ as $R_{P_{r}}=\sum_{j=1}^{24} A_{j}$, where

$$
\begin{aligned}
& A_{1}=\frac{4 \mathrm{~K}_{\overline{1}}^{3} \mathrm{~K}_{1}^{3}}{\mathrm{~K}^{6} \mathrm{~S}^{3}}, \\
& A_{2}=-\frac{2 \mathrm{~K}_{\overline{1}} \mathrm{~K}_{\overline{1} \overline{1}} \mathrm{~K}_{1}{ }^{3}}{K^{5} \mathrm{~S}^{3}}, \\
& A_{3}=-\frac{8 \mathrm{~K}_{\overline{1}}^{2} \mathrm{~K}_{1}^{2} \mathrm{~K}_{1 \overline{1}}}{\mathrm{~K}^{5} \mathrm{~S}^{3}}, \\
& A_{4}=\frac{2 \mathrm{~K}_{\overline{1} \overline{1}} \mathrm{~K}_{1}^{2} \mathrm{~K}_{1 \overline{1}}}{\mathrm{~K}^{4} \mathrm{~S}^{3}}, \\
& A_{5}=\frac{4 \mathrm{~K}_{\overline{1}} \mathrm{~K}_{1} \mathrm{~K}_{1 \overline{1}}^{2}}{\mathrm{~K}^{4} \mathrm{~S}^{3}}, \\
& A_{6}=\frac{6 \mathrm{~K}_{\overline{1}}^{2} \mathrm{~K}_{1}^{2}}{\mathrm{~K}^{4} \mathrm{~S}^{2}}, \\
& A_{7}=-\frac{2 \mathrm{~K}_{\overline{1} \overline{1}} \mathrm{~K}_{1}^{2}}{\mathrm{~K}^{3} \mathrm{~S}^{2}} \text {, } \\
& A_{8}=-\frac{8 \mathrm{~K}_{\overline{1}} \mathrm{~K}_{1} \mathrm{~K}_{1 \overline{1}}}{\mathrm{~K}^{3} \mathrm{~S}^{2}} \text {, } \\
& A_{9}=\frac{2 \mathrm{~K}_{1 \overline{1}}^{2}}{\mathrm{~K}^{2} \mathrm{~S}^{2}}, \\
& A_{10}=\frac{2 \mathrm{~K}_{\overline{1}} \mathrm{~K}_{1}^{2} \mathrm{~K}_{1 \overline{1} \overline{1}}}{\mathrm{~K}^{4} \mathrm{~S}^{3}} \\
& A_{11}=-\frac{2 \mathrm{~K}_{1} \mathrm{~K}_{1 \overline{1}} \mathrm{~K}_{1 \overline{1} \overline{1}}}{\mathrm{~K}^{3} \mathrm{~S}^{3}} \text {, } \\
& A_{12}=\frac{2 \mathrm{~K}_{1} \mathrm{~K}_{1 \overline{1} \overline{1}}}{\mathrm{~K}^{2} \mathrm{~S}^{2}} \\
& A_{13}=-\frac{2 \mathrm{~K}_{\overline{1}}^{3} \mathrm{~K}_{1} \mathrm{~K}_{11}}{\mathrm{~K}^{5} \mathrm{~S}^{3}}, \\
& A_{14}=\frac{\mathrm{K}_{\overline{1}} \mathrm{~K}_{\overline{1} \overline{1}} \mathrm{~K}_{1} \mathrm{~K}_{11}}{\mathrm{~K}^{4} \mathrm{~S}^{3}}, \\
& A_{15}=\frac{2 \mathrm{~K}_{\overline{1}}^{2} \mathrm{~K}_{1 \overline{1}} \mathrm{~K}_{11}}{\mathrm{~K}^{4} \mathrm{~S}^{3}} \text {, } \\
& A_{16}=-\frac{2 \mathrm{~K}_{\overline{1}}^{2} \mathrm{~K}_{11}}{\mathrm{~K}^{3} \mathrm{~S}^{2}}, \quad A_{17}=\frac{\mathrm{K}_{11} \mathrm{~K}_{\overline{1} \overline{1}}}{\mathrm{~K}^{2} \mathrm{~S}^{2}}, \\
& A_{18}=-\frac{\mathrm{K}_{\overline{1}} \mathrm{~K}_{1 \overline{1} \overline{1}} \mathrm{~K}_{11}}{\mathrm{~K}^{3} \mathrm{~S}^{3}} \text {, } \\
& A_{19}=\frac{2 \mathrm{~K}_{\overline{1}}^{2} \mathrm{~K}_{1} \mathrm{~K}_{11 \overline{1}}}{\mathrm{~K}^{4} \mathrm{~S}^{3}} \\
& A_{20}=-\frac{\mathrm{K}_{\overline{1} \overline{1}} \mathrm{~K}_{1} \mathrm{~K}_{11 \overline{1}}}{\mathrm{~K}^{3} \mathrm{~S}^{3}}, \\
& A_{21}=-\frac{2 \mathrm{~K}_{\overline{1}} \mathrm{~K}_{1 \overline{1}} \mathrm{~K}_{11 \overline{1}}}{\mathrm{~K}^{3} \mathrm{~S}^{3}}, \\
& A_{22}=\frac{2 \mathrm{~K}_{\overline{1}} \mathrm{~K}_{11 \overline{1}}}{\mathrm{~K}^{2} \mathrm{~S}^{2}}, \\
& A_{23}=\frac{\mathrm{K}_{1 \overline{1} \overline{1}} \mathrm{~K}_{11 \overline{1}}}{\mathrm{~K}^{2} \mathrm{~S}^{3}} \\
& A_{24}=-\frac{\mathrm{K}_{11 \overline{1} \overline{1}}}{\mathrm{~K} \mathrm{~S}^{2}} \text {. }
\end{aligned}
$$


By direct computation one obtains

$$
\begin{aligned}
& \mathrm{K}=-\frac{1}{\pi|z|^{2} \log \left(r^{2}\right)}+\pi^{-1} \sum_{j=0}^{\infty}\left(\frac{r^{2+2 j}}{\left(-r^{2+2 j}+|z|^{2}\right)^{2}}+\frac{r^{2 j}}{\left(1-r^{2 j}|z|^{2}\right)^{2}}\right), \\
& \mathrm{K}_{1}=\frac{1}{\pi z|z|^{2} \log \left(r^{2}\right)}+\pi^{-1} \sum_{j=0}^{\infty}\left(-\frac{2 r^{2+2 j} \bar{z}}{\left(-r^{2+2 j}+|z|^{2}\right)^{3}}+\frac{2 r^{4 j} \bar{z}}{\left(1-r^{2 j}|z|^{2}\right)^{3}}\right), \\
& \mathrm{K}_{\overline{1}}=\overline{\mathrm{K}_{1}} \text {, } \\
& \mathrm{K}_{11}=-\frac{2}{\pi z^{2}|z|^{2} \log \left(r^{2}\right)}+\pi^{-1} \sum_{j=0}^{\infty}\left(\frac{6 r^{2+2 j} \bar{z}^{2}}{\left(-r^{2+2 j}+|z|^{2}\right)^{4}}+\frac{6 r^{6 j} \bar{z}^{2}}{\left(1-r^{2 j}|z|^{2}\right)^{4}}\right), \\
& \mathrm{K}_{\overline{1} \overline{1}}=\overline{\mathrm{K}_{11}} \text {, } \\
& \mathrm{K}_{1 \overline{1}}=-\frac{1}{\pi|z|^{4} \log \left(r^{2}\right)}+\pi^{-1} \sum_{j=0}^{\infty}\left(\frac{6 r^{2+2 j}|z|^{2}}{\left(-r^{2+2 j}+|z|^{2}\right)^{4}}-\frac{2 r^{2+2 j}}{\left(-r^{2+2 j}+|z|^{2}\right)^{3}}\right. \\
& \left.+\frac{6 r^{6 j}|z|^{2}}{\left(1-r^{2 j}|z|^{2}\right)^{4}}+\frac{2 r^{4 j}}{\left(1-r^{2 j}|z|^{2}\right)^{3}}\right) \\
& \mathrm{K}_{11 \overline{1}}=\frac{2}{\pi z|z|^{4} \log \left(r^{2}\right)}+\pi^{-1} \sum_{j=0}^{\infty}\left(-\frac{24 r^{2+2 j}|z|^{2} \bar{z}}{\left(-r^{2+2 j}+|z|^{2}\right)^{5}}+\frac{12 r^{2+2 j} \bar{z}}{\left(-r^{2+2 j}+|z|^{2}\right)^{4}}\right. \\
& \left.+\frac{24 r^{8 j}|z|^{2} \bar{z}}{\left(1-r^{2 j}|z|^{2}\right)^{5}}+\frac{12 r^{6 j} \bar{z}}{\left(1-r^{2 j}|z|^{2}\right)^{4}}\right) \\
& \mathrm{K}_{1 \overline{1} \overline{1}}=\overline{\mathrm{K}_{11 \overline{1}}} \text {, } \\
& \mathrm{K}_{11 \overline{1} \overline{1}}=-\frac{4}{\pi|z|^{6} \log \left(r^{2}\right)}+\pi^{-1} \sum_{j=0}^{\infty}\left[6 r ^ { 2 + 2 j } \left(\frac{20|z|^{4}}{\left(-r^{2+2 j}+|z|^{2}\right)^{6}}\right.\right. \\
& \left.-\frac{16|z|^{2}}{\left(-r^{2+2 j}+|z|^{2}\right)^{5}}+\frac{2}{\left(-r^{2+2 j}+|z|^{2}\right)^{4}}\right) \\
& \left.+6 r^{6 j}\left(\frac{20 r^{4 j}|z|^{4}}{\left(1-r^{2 j}|z|^{2}\right)^{6}}+\frac{16 r^{2 j}|z|^{2}}{\left(1-r^{2 j}|z|^{2}\right)^{5}}+\frac{2}{\left(1-r^{2 j}|z|^{2}\right)^{4}}\right)\right] \text {. }
\end{aligned}
$$

For the special choice $z=\sqrt{r} \in \mathbb{R}_{+}$one obtains

$$
\begin{aligned}
\mathrm{K} & =-\frac{1}{\pi r \log \left(r^{2}\right)}+\pi^{-1} \sum_{j=0}^{\infty}\left(\frac{r^{2 j}}{\left(-r^{1+2 j}+1\right)^{2}}+\frac{r^{2 j}}{\left(1-r^{2 j+1}\right)^{2}}\right), \\
\mathrm{K}_{1} & =\mathrm{K}_{\overline{1}}=\frac{1}{\pi r^{3 / 2} \log \left(r^{2}\right)}+\pi^{-1} \sum_{j=0}^{\infty}\left(\frac{2 r^{1 / 2+4 j}}{\left(-r^{1+2 j}+1\right)^{3}}-\frac{2 r^{-1 / 2+2 j}}{\left(1-r^{2 j+1}\right)^{3}}\right) \\
\mathrm{K}_{11} & =\mathrm{K}_{\overline{1} \overline{1}}=-\frac{2}{\pi r^{2} \log \left(r^{2}\right)}+\pi^{-1} \sum_{j=0}^{\infty}\left(\frac{6 r^{1+2 j}}{\left(-r^{1+2 j}+1\right)^{4}}+\frac{6 r^{2 j-1}}{\left(1-r^{2 j+1}\right)^{4}}\right)
\end{aligned}
$$




$$
\begin{gathered}
\mathrm{K}_{1 \overline{1}}=-\frac{1}{\pi r^{2} \log \left(r^{2}\right)}+\pi^{-1} \sum_{j=0}^{\infty}\left(\frac{6 r^{1+6 j}}{\left(-r^{1+2 j}+1\right)^{4}}+\frac{2 r^{4 j}}{\left(-r^{1+2 j}+1\right)^{3}}\right. \\
\left.\quad+\frac{6 r^{2 j-1}}{\left(1-r^{1+2 j}\right)^{4}}-\frac{2 r^{2 j-1}}{\left(1-r^{2 j+1}\right)^{3}}\right), \\
\mathrm{K}_{11 \overline{1}}=\mathrm{K}_{1 \overline{1} \overline{1}}=\frac{2}{\pi r^{5 / 2} \log \left(r^{2}\right)}+\pi^{-1} \sum_{j=0}^{\infty}\left(\frac{24 r^{3 / 2+8 j}}{\left(-r^{1+2 j}+1\right)^{5}}+\frac{12 r^{1 / 2+6 j}}{\left(-r^{1+2 j+1)^{4}}\right.}\right. \\
\mathrm{K}_{11 \overline{1} \overline{1}=}-\frac{4}{\pi r^{3} \log \left(r^{2}\right)}+\pi^{-1} \sum_{j=0}^{\infty}\left(\frac{120 r^{10 j+2}}{\left(-r^{1+2 j}+1\right)^{6}+\frac{96 r^{1+3 / 2}}{\left(-r^{1+2 j}+1\right)^{5}}}+\frac{12 r^{2 j-3 / 2}}{\left(1-r^{2 j+1}\right)^{4}}\right), \\
\left.+\frac{12 r^{6 j}}{\left(-r^{1+2 j}+1\right)^{4}}\right)+\left(\frac{120 r^{2 j-2}}{\left(1-r^{2 j+1}\right)^{6}}-\frac{96 r^{2 j-2}}{\left(1-r^{2 j+1}\right)^{5}}+\frac{12 r^{2 j-2}}{\left(1-r^{2 j+1}\right)^{4}}\right) .
\end{gathered}
$$

All of the above series are locally uniformly convergent in the unit disc and the summands are of the form $f(\sqrt{r})$ with $f$ real-analytic (with exception of the very first summands which may contribute some singular terms). Therefore each of the above expressions is of the form $F(\sqrt{r})+$ singular part, with $F$ real-analytic, hence one can write:

$$
\begin{aligned}
\mathrm{K} & =-\frac{1}{\pi r \log \left(r^{2}\right)}+\pi^{-1}\left(2+4 r+8 r^{2}+O\left(r^{3}\right)\right), \\
\mathrm{K}_{1} & =\mathrm{K}_{\overline{1}}=\frac{1}{\pi r^{3 / 2} \log \left(r^{2}\right)}+\pi^{-1}\left(\frac{-2}{\sqrt{r}}-4 \sqrt{r}-8 r^{3 / 2}+O\left(r^{5 / 2}\right)\right), \\
\mathrm{K}_{11} & =\mathrm{K}_{\overline{1} \overline{1}}=-\frac{2}{\pi r^{2} \log \left(r^{2}\right)}+\pi^{-1}\left(\frac{6}{r}+24+O(r)\right), \\
\mathrm{K}_{1 \overline{1}} & =-\frac{1}{\pi r^{2} \log \left(r^{2}\right)}+\pi^{-1}\left(\frac{4}{r}+20+64 r+O\left(r^{2}\right)\right), \\
\mathrm{K}_{11 \overline{1}} & =\mathrm{K}_{1 \overline{1} \overline{1}}=\frac{2}{\pi r^{5 / 2} \log \left(r^{2}\right)}+\pi^{-1}\left(-\frac{12}{r^{3 / 2}}-\frac{72}{\sqrt{r}}+O\left(r^{1 / 2}\right)\right), \\
\mathrm{K}_{11 \overline{1} \overline{1}} & =-\frac{4}{\pi r^{3} \log \left(r^{2}\right)}+\pi^{-1}\left(\frac{36}{r^{2}}+\frac{288}{r}+O(C)\right),
\end{aligned}
$$

where as usual $O\left(r^{\alpha}\right)$ is a substitute for an expression which divided by $r^{\alpha}$ is bounded when $r$ tends to $0, r>0$. Note that in each representation above the powers of $r$ in the terms $\frac{1}{\pi r^{\alpha} \log \left(r^{2}\right)}$ and $O\left(r^{\beta}\right)$ satisfy

$$
\alpha+\beta \geq 3 \text {. }
$$

Our first task is to show that one can get rid of the $O$ 's in the expressions representing $A_{j}(r), j=1, \ldots, 24$, in the above notation. Let $A_{j}^{*}(r)$ be $A_{j}(r)$ with the $O$ 's deleted. 
One has to show that $\lim _{r \rightarrow 0^{+}}\left(A_{j}(r)-A_{j}^{*}(r)\right)=0, j=1, \ldots, 24$.

After some elementary algebraic manipulations one obtains

$$
\begin{aligned}
& A_{j}(r)= \\
& \frac{ \pm 1}{\left(r \log \left(r^{2}\right)\right)^{p_{j}}} \frac{\prod_{i=1}^{m_{j}}\left(a_{0}^{i j}+a_{1}^{i j} r^{\alpha_{1}^{i j}} \log \left(r^{2}\right)+\cdots+a_{k(i j)}^{i j} r^{\alpha_{k(i j)}^{i j}} \log \left(r^{2}\right) O\left(r^{\gamma_{k(i j)}^{i j}}\right)\right)}{\prod_{i=1}^{n_{j}}\left(b_{0}^{i j}+b_{1}^{i j} r^{\beta_{1}^{i j}} \log \left(r^{2}\right)+\cdots+b_{s(i j)}^{i j} r_{s(i j)}^{\beta_{s j}} \log \left(r^{2}\right) O\left(r_{s(i j)}^{\delta^{i j}}\right)\right)} .
\end{aligned}
$$

Here $p_{j}=2$ for $j=6,7,8,9,12,16,17,22,24$, otherwise $p_{j}=3$, and the numbers $\alpha_{l}^{i j}$ and $\beta_{l}^{i j}$ form ascending (with respect to $l$ ) sequences of $k(i j)$, respectively $s(i j)$ positive rational numbers. The notation $k(i j)$ and $s(i j)$ is to stress the fact that the length of each sequence depends on both $i$ and $j$. The $a_{l}^{i j}$ and $b_{l}^{i j}$ are some (rational) constants. Finally $\alpha_{k(i j)}^{i j}+\gamma_{k(i j)}^{i j} \geq 3$ and $\beta_{s(i j)}^{i j}+\delta_{s(i j)}^{i j} \geq 3$ (by $(\dagger)$, the reason why $\alpha+\beta$ is 4 , not 3 in the expansions of $\mathrm{K}, \mathrm{K}_{1}, \mathrm{~K}_{1 \overline{1}}$ is that the lowest power of $r$ disappears when one manipulates $\left.-\frac{\mathrm{K}_{\overline{1}} \mathrm{~K}_{1}}{\mathrm{~K}^{2}}+\frac{\mathrm{K}_{1 \overline{1}}}{\mathrm{~K}}\right)$. In what follows we shorten $k(i j)$ and $s(i j)$ to just $k$ and $s$ but keep in mind that they depend on both $i$ and $j$.

The numerator of $A_{j}(r)-A_{j}^{*}(r)$ is

$$
\begin{aligned}
& \prod_{i=1}^{m_{j}}\left(a_{0}^{i j}+\sum_{l=1}^{k-1} a_{l}^{i j} r^{\alpha_{l}^{i j}} \log \left(r^{2}\right)+a_{k}^{i j} r^{\alpha_{k}^{i j}}\right.\left.\log \left(r^{2}\right) O\left(r^{\gamma_{k}^{i j}}\right)\right) \\
& \times \prod_{i=1}^{n_{j}}\left(b_{0}^{i j}+\sum_{l=1}^{s-1} b_{l}^{i j} r^{\beta_{l}^{i j}} \log \left(r^{2}\right)\right) \\
&-\prod_{i=1}^{n_{j}}\left(b_{0}^{i j}+\sum_{l=1}^{s-1} b_{l}^{i j} r^{\beta_{l}^{i j}} \log \left(r^{2}\right)+b_{s}^{i j} r^{\beta_{s}^{i j}} \log \left(r^{2}\right) O\left(r^{\delta_{s}^{i j}}\right)\right) \\
& \times \prod_{i=1}^{m_{j}}\left(a_{0}^{i j}+\sum_{l=1}^{k-1} a_{l}^{i j} r^{\alpha_{l}^{i j}} \log \left(r^{2}\right)\right)
\end{aligned}
$$

and we see that all the terms not containing an $O$ kill each other. What remains is $O\left(r^{3} \log (r)\right)$ (by $\left.(\dagger)\right)$.

The denominator is

$$
\begin{aligned}
&\left(r \log \left(r^{2}\right)\right)^{p_{j}} \prod_{i=1}^{n_{j}}\left(b_{0}^{i j}+\sum_{l=1}^{s-1} b_{l}^{i j} r^{\beta_{l}^{i j}} \log \left(r^{2}\right)+b_{s}^{i j} r^{\beta_{s}^{i j}} \log \left(r^{2}\right) O\left(r^{\delta_{s}^{i j}}\right)\right) \\
& \times \prod_{i=1}^{n_{j}}\left(b_{0}^{i j}+\sum_{l=1}^{s-1} b_{l}^{i j} r^{\beta_{l}^{i j}} \log \left(r^{2}\right)\right),
\end{aligned}
$$

which is $O\left(\left(r \log \left(r^{2}\right)\right)^{p_{j}}\right)$ (no $b_{0}^{i j}$ is zero), and hence the ratio tends to 0 . 
The terms $A_{j}^{*}$ can be asymptotically evaluated as follows:

$$
\begin{aligned}
& A_{23}^{*}(r) \approx-\frac{1}{2 r^{3}\left(\log \left(r^{2}\right)\right)^{3}}+\frac{7}{r^{2}\left(\log \left(r^{2}\right)\right)^{2}}+\frac{12}{r^{2}\left(\log \left(r^{2}\right)\right)^{3}}-\frac{30}{r \log \left(r^{2}\right)}, \\
& A_{21}^{*}(r) \approx \frac{1}{2 r^{3}\left(\log \left(r^{2}\right)\right)^{3}}-\frac{6}{r^{2}\left(\log \left(r^{2}\right)\right)^{2}}-\frac{12}{r^{2}\left(\log \left(r^{2}\right)\right)^{3}}+\frac{22}{r \log \left(r^{2}\right)}, \\
& A_{20}^{*}(r) \approx \frac{1}{2 r^{3}\left(\log \left(r^{2}\right)\right)^{3}}-\frac{11}{2 r^{2}\left(\log \left(r^{2}\right)\right)^{2}}-\frac{12}{r^{2}\left(\log \left(r^{2}\right)\right)^{3}}+\frac{18}{r \log \left(r^{2}\right)}, \\
& A_{19}^{*}(r) \approx-\frac{1}{2 r^{3}\left(\log \left(r^{2}\right)\right)^{3}}+\frac{5}{r^{2}\left(\log \left(r^{2}\right)\right)^{2}}+\frac{12}{r^{2}\left(\log \left(r^{2}\right)\right)^{3}}-\frac{14}{r \log \left(r^{2}\right)}, \\
& A_{18}^{*}(r) \approx \frac{1}{2 r^{3}\left(\log \left(r^{2}\right)\right)^{3}}-\frac{11}{2 r^{2}\left(\log \left(r^{2}\right)\right)^{2}}-\frac{12}{r^{2}\left(\log \left(r^{2}\right)\right)^{3}}+\frac{18}{r \log \left(r^{2}\right)}, \\
& A_{15}^{*}(r) \approx-\frac{1}{2 r^{3}\left(\log \left(r^{2}\right)\right)^{3}}+\frac{9}{2 r^{2}\left(\log \left(r^{2}\right)\right)^{2}}+\frac{12}{r^{2}\left(\log \left(r^{2}\right)\right)^{3}}-\frac{13}{r \log \left(r^{2}\right)}, \\
& A_{14}^{*}(r) \approx-\frac{1}{2 r^{3}\left(\log \left(r^{2}\right)\right)^{3}}+\frac{4}{r^{2}\left(\log \left(r^{2}\right)\right)^{2}}+\frac{12}{r^{2}\left(\log \left(r^{2}\right)\right)^{3}}-\frac{21}{2 r \log \left(r^{2}\right)}, \\
& A_{13}^{*}(r) \approx \frac{1}{2 r^{3}\left(\log \left(r^{2}\right)\right)^{3}}-\frac{7}{2 r^{2}\left(\log \left(r^{2}\right)\right)^{2}}-\frac{12}{r^{2}\left(\log \left(r^{2}\right)\right)^{3}}+\frac{8}{r \log \left(r^{2}\right)}, \\
& A_{11}^{*}(r) \approx \frac{1}{2 r^{3}\left(\log \left(r^{2}\right)\right)^{3}}-\frac{6}{r^{2}\left(\log \left(r^{2}\right)\right)^{2}}-\frac{12}{r^{2}\left(\log \left(r^{2}\right)\right)^{3}}+\frac{22}{r \log \left(r^{2}\right)}, \\
& A_{10}^{*}(r) \approx-\frac{1}{2 r^{3}\left(\log \left(r^{2}\right)\right)^{3}}+\frac{5}{r^{2}\left(\log \left(r^{2}\right)\right)^{2}}+\frac{12}{r^{2}\left(\log \left(r^{2}\right)\right)^{3}}-\frac{14}{r \log \left(r^{2}\right)}, \\
& A_{5}^{*}(r) \approx-\frac{1}{2 r^{3}\left(\log \left(r^{2}\right)\right)^{3}}+\frac{5}{r^{2}\left(\log \left(r^{2}\right)\right)^{2}}+\frac{12}{r^{2}\left(\log \left(r^{2}\right)\right)^{3}}-\frac{16}{r \log \left(r^{2}\right)}, \\
& A_{4}^{*}(r) \approx-\frac{1}{2 r^{3}\left(\log \left(r^{2}\right)\right)^{3}}+\frac{9}{2 r^{2}\left(\log \left(r^{2}\right)\right)^{2}}+\frac{12}{r^{2}\left(\log \left(r^{2}\right)\right)^{3}}-\frac{13}{r \log \left(r^{2}\right)}, \\
& A_{3}^{*}(r) \approx \frac{1}{r^{3}\left(\log \left(r^{2}\right)\right)^{3}}-\frac{8}{r^{2}\left(\log \left(r^{2}\right)\right)^{2}}-\frac{24}{r^{2}\left(\log \left(r^{2}\right)\right)^{3}}+\frac{20}{r \log \left(r^{2}\right)}, \\
& A_{2}^{*}(r) \approx \frac{1}{2 r^{3}\left(\log \left(r^{2}\right)\right)^{3}}-\frac{7}{2 r^{2}\left(\log \left(r^{2}\right)\right)^{2}}-\frac{12}{r^{2}\left(\log \left(r^{2}\right)\right)^{3}}+\frac{8}{r \log \left(r^{2}\right)}, \\
& A_{1}^{*}(r) \approx-\frac{1}{2 r^{3}\left(\log \left(r^{2}\right)\right)^{3}}+\frac{3}{r^{2}\left(\log \left(r^{2}\right)\right)^{2}}+\frac{12}{r^{2}\left(\log \left(r^{2}\right)\right)^{3}}-\frac{6}{r \log \left(r^{2}\right)},
\end{aligned}
$$

where

$$
A_{j}^{*}(r) \approx \frac{a_{j}}{r^{3}\left(\log \left(r^{2}\right)\right)^{3}}+\frac{b_{j}}{r^{2}\left(\log \left(r^{2}\right)\right)^{2}}+\frac{c_{j}}{r^{2}\left(\log \left(r^{2}\right)\right)^{3}}+\frac{d_{j}}{r \log \left(r^{2}\right)}
$$

should be read as

$\lim _{r \rightarrow 0^{+}} A_{j}^{*}(r) r^{3}\left(\log \left(r^{2}\right)\right)^{3}=a_{j}, \quad \lim _{r \rightarrow 0^{+}}\left(A_{j}^{*}(r)-\frac{a_{j}}{r^{3}\left(\log \left(r^{2}\right)\right)^{3}}\right) r^{2}\left(\log \left(r^{2}\right)\right)^{2}=b_{j}$, 


$$
\begin{gathered}
\lim _{r \rightarrow 0^{+}}\left(A_{j}^{*}(r)-\frac{a_{j}}{r^{3}\left(\log \left(r^{2}\right)\right)^{3}}-\frac{b_{j}}{r^{2}\left(\log \left(r^{2}\right)\right)^{2}}\right) r^{2}\left(\log \left(r^{2}\right)\right)^{3}=c_{j}, \\
\lim _{r \rightarrow 0^{+}}\left(A_{j}^{*}(r)-\frac{a_{j}}{r^{3}\left(\log \left(r^{2}\right)\right)^{3}}-\frac{b_{j}}{r^{2}\left(\log \left(r^{2}\right)\right)^{2}}-\frac{c_{j}}{r^{2}\left(\log \left(r^{2}\right)\right)^{3}}\right) r \log \left(r^{2}\right)=d_{j},
\end{gathered}
$$

and

$$
\begin{aligned}
& A_{24}^{*}(r) \approx \frac{1}{r^{2}\left(\log \left(r^{2}\right)\right)^{2}}+\frac{11}{r \log \left(r^{2}\right)}, \\
& A_{22}^{*}(r) \approx-\frac{1}{r^{2}\left(\log \left(r^{2}\right)\right)^{2}}-\frac{8}{r \log \left(r^{2}\right)}, \\
& A_{17}^{*}(r) \approx-\frac{1}{2 r^{2}\left(\log \left(r^{2}\right)\right)^{2}}-\frac{6}{r \log \left(r^{2}\right)}, \\
& A_{16}^{*}(r) \approx \frac{1}{2 r^{2}\left(\log \left(r^{2}\right)\right)^{2}}+\frac{5}{r \log \left(r^{2}\right)}, \\
& A_{12}^{*}(r) \approx-\frac{1}{2 r^{2}\left(\log \left(r^{2}\right)\right)^{2}}-\frac{8}{r \log \left(r^{2}\right)}, \\
& A_{9}^{*}(r) \approx-\frac{1}{2 r^{2}\left(\log \left(r^{2}\right)\right)^{2}}-\frac{4}{r \log \left(r^{2}\right)}, \\
& A_{8}^{*}(r) \approx \frac{2}{r^{2}\left(\log \left(r^{2}\right)\right)^{2}}+\frac{12}{r \log \left(r^{2}\right)}, \\
& A_{7}^{*}(r) \approx \frac{1}{2 r^{2}\left(\log \left(r^{2}\right)\right)^{2}}+\frac{5}{r \log \left(r^{2}\right)}, \\
& A_{6}^{*}(r) \approx-\frac{3}{2 r^{2}\left(\log \left(r^{2}\right)\right)^{2}}-\frac{6}{r \log \left(r^{2}\right)},
\end{aligned}
$$

where

$$
A_{j}^{*}(r) \approx \frac{a_{j}}{r^{2}\left(\log \left(r^{2}\right)\right)^{2}}+\frac{b_{j}}{r \log \left(r^{2}\right)}
$$

should be read as

$$
\lim _{r \rightarrow 0^{+}} A_{j}^{*}(r) r^{2}\left(\log \left(r^{2}\right)\right)^{2}=a_{j}, \quad \lim _{r \rightarrow 0^{+}}\left(A_{j}^{*}(r)-\frac{a_{j}}{r^{2}\left(\log \left(r^{2}\right)\right)^{2}}\right) r \log \left(r^{2}\right)=b_{j} .
$$

Hence

$$
\begin{gathered}
\sum_{j=1}^{24} A_{j}^{*}(r) \approx \frac{0}{r^{3}\left(\log \left(r^{2}\right)\right)^{3}}+\frac{0}{r^{2}\left(\log \left(r^{2}\right)\right)^{2}}+\frac{0}{r^{2}\left(\log \left(r^{2}\right)\right)^{3}}+\frac{1}{2 r \log \left(r^{2}\right)}, \\
\lim _{r \rightarrow 0^{+}} \sum_{j=1}^{24} A_{j}(r)=\lim _{r \rightarrow 0^{+}} \sum_{j=1}^{24} A_{j}^{*}(r)=-\infty
\end{gathered}
$$

This proves the first part of Theorem 0.1 . 
For the special choice $z=r^{3 / 10} \in \mathbb{R}_{+}$one has

$$
\begin{aligned}
& \mathrm{K}=-\frac{1}{\pi r^{3 / 5} \log \left(r^{2}\right)}+\pi^{-1} \sum_{j=0}^{\infty}\left(\frac{r^{2 j}}{\left(-r^{3 / 5+2 j}+1\right)^{2}}+\frac{r^{2 j+4 / 5}}{\left(1-r^{2 j+7 / 5}\right)^{2}}\right) \\
& \mathrm{K}_{1}=\frac{1}{\pi r^{9 / 10} \log \left(r^{2}\right)}+\pi^{-1} \sum_{j=0}^{\infty}\left(\frac{2 r^{3 / 10+4 j}}{\left(-r^{3 / 5+2 j}+1\right)^{3}}-\frac{2 r^{1 / 2+2 j}}{\left(1-r^{2 j+7 / 5}\right)^{3}}\right) \\
& \mathrm{K}_{\overline{1}}=\mathrm{K}_{1}, \\
& \mathrm{~K}_{11}=-\frac{2}{\pi r^{6 / 5} \log \left(r^{2}\right)}+\pi^{-1} \sum_{j=0}^{\infty}\left(\frac{6 r^{3 / 5+6 j}}{\left(-r^{3 / 5+2 j}+1\right)^{4}}+\frac{6 r^{1 / 5+2 j}}{\left(1-r^{2 j+7 / 5}\right)^{4}}\right) \\
& \mathrm{K}_{\overline{1} \overline{1}}=\mathrm{K}_{11}, \quad \frac{1}{\pi r^{6 / 5} \log \left(r^{2}\right)}+\pi^{-1} \sum_{j=0}^{\infty}\left[\frac{6 r^{3 / 5+6 j}}{\left(-r^{3 / 5+2 j}+1\right)^{4}}+\frac{2 r^{4 j}}{\left(-r^{3 / 5+2 j}+1\right)^{3}}\right. \\
& \mathrm{K}_{1 \overline{1}}\left.=-\frac{2 r^{2 j+1 / 5}}{\left(1-r^{2 j+7 / 5}\right)^{3}}\right] \\
& \mathrm{K}_{11 \overline{1}}=\frac{2}{\pi r^{3 / 2} \log \left(r^{2}\right)}+\pi^{-1} \sum_{j=0}^{\infty}\left[\frac{24 r^{9 / 10+8 j}}{\left(-r^{3 / 5+2 j}+1\right)^{5}}+\frac{12 r^{3 / 10+6 j}}{\left(-r^{3 / 5+2 j}+1\right)^{4}}\right. \\
& \mathrm{K}_{1 \overline{1} \overline{1}}\left.=\mathrm{K}_{11 \overline{1}}, \quad \frac{24 r^{2 j-1 / 10}}{\left(1-r^{7 / 5+2 j}\right)^{5}}+\frac{12 r^{2 j-1 / 10}}{\left(1-r^{2 j+7 / 5}\right)^{4}}\right] \\
& \mathrm{K}_{11 \overline{1} \overline{1}}=-\frac{4}{\pi r^{9 / 5} \log \left(r^{2}\right)}+\pi^{-1} \sum_{j=0}^{\infty}\left[\frac{120 r^{10 j+6 / 5}}{\left(-r^{3 / 5+2 j}+1\right)^{6}}+\frac{96 r^{3 / 5+8 j}}{\left(-r^{3 / 5+2 j}+1\right)^{5}}\right. \\
&\left.+\frac{12 r^{6 j}}{\left(-r^{3 / 5+2 j}+1\right)^{4}}+\frac{120 r^{2 j-2 / 5}}{\left(1-r^{2 j+7 / 5}\right)^{6}}-\frac{96 r^{2 j-2 / 5}}{\left(1-r^{2 j+7 / 5}\right)^{5}}+\frac{12 r^{2 j-2 / 5}}{\left(1-r^{2 j+7 / 5}\right)^{4}}\right]
\end{aligned}
$$

As above each sum is of the form $G\left(r^{1 / 10}\right)+$ singular part, with $G$ realanalytic. Now

$$
\begin{aligned}
\mathrm{K} & =-\frac{1}{\pi r^{3 / 5} \log \left(r^{2}\right)}+\pi^{-1}\left(1+2 r^{3 / 5}+r^{4 / 5}+3 r^{6 / 5}+O\left(r^{9 / 5}\right)\right), \\
\mathrm{K}_{1} & =\mathrm{K}_{\overline{1}}=\frac{1}{\pi r^{9 / 10} \log \left(r^{2}\right)}+\pi^{-1}\left(2 r^{3 / 10}-2 r^{1 / 2}+6 r^{9 / 10}+O\left(r^{3 / 2}\right)\right), \\
\mathrm{K}_{11} & =\mathrm{K}_{\overline{1} \overline{1}}=-\frac{2}{\pi r^{6 / 5} \log \left(r^{2}\right)}+\pi^{-1}\left(6 r^{1 / 5}+O\left(r^{3 / 5}\right)\right), \\
\mathrm{K}_{1 \overline{1}} & =-\frac{1}{\pi r^{6 / 5} \log \left(r^{2}\right)}+\pi^{-1}\left(2+4 r^{1 / 5}+12 r^{3 / 5}+O\left(r^{6 / 5}\right)\right),
\end{aligned}
$$




$$
\begin{aligned}
\mathrm{K}_{11 \overline{1}} & =\mathrm{K}_{1 \overline{1} \overline{1}}=\frac{2}{\pi r^{3 / 2} \log \left(r^{2}\right)}+\pi^{-1}\left(-\frac{12}{r^{1 / 10}}+O\left(r^{3 / 10}\right)\right), \\
\mathrm{K}_{11 \overline{1} \overline{1}} & =-\frac{4}{\pi r^{9 / 5} \log \left(r^{2}\right)}+\pi^{-1}\left(\frac{36}{r^{2 / 5}}+O(C)\right) .
\end{aligned}
$$

This time the goal is to have

$$
\alpha+\beta \geq 9 / 5
$$

for $\alpha$ and $\beta$ being the powers of $r$ in $1 /\left(\pi r^{\alpha} \log \left(r^{2}\right)\right)$ and $O\left(r^{\beta}\right)$ respectively. The argument with passing from $A_{j}(r)$ to $A_{j}^{*}(r)$ is almost the same, one only has to adjust the terms $\left(r \log \left(r^{2}\right)\right)^{p_{j}}$ (the correct ones are now $\left.r^{p_{j}} \log \left(r^{2}\right)^{\frac{5}{3}} p_{j}\right)$ and consider new $p_{j}$ 's, namely $p_{j}=6 / 5$, for $j=6,7,8,9,12,16,17,22,24$ and $p_{j}=9 / 5$ otherwise.

Now the asymptotic expansions of $A_{j}^{*}(r)$ are:

$$
\begin{aligned}
& A_{23}^{*}(r) \approx-\frac{4}{r^{9 / 5}\left(\log \left(r^{2}\right)\right)^{3}}+\frac{16}{r^{6 / 5}\left(\log \left(r^{2}\right)\right)^{2}}+\frac{96}{r^{6 / 5}\left(\log \left(r^{2}\right)\right)^{3}}+\frac{12}{r\left(\log \left(r^{2}\right)\right)^{3}} \\
& -\frac{24}{r^{3 / 5} \log \left(r^{2}\right)}-\frac{376}{r^{3 / 5}\left(\log \left(r^{2}\right)\right)^{2}}-\frac{1212}{r^{3 / 5}\left(\log \left(r^{2}\right)\right)^{3}}-\frac{32}{r^{2 / 5}\left(\log \left(r^{2}\right)\right)^{2}} \\
& -\frac{384}{r^{2 / 5}\left(\log \left(r^{2}\right)\right)^{3}}-\frac{24}{r^{1 / 5}\left(\log \left(r^{2}\right)\right)^{3}}+16 \\
& A_{21}^{*}(r) \approx \frac{4}{r^{9 / 5}\left(\log \left(r^{2}\right)\right)^{3}}-\frac{12}{r^{6 / 5}\left(\log \left(r^{2}\right)\right)^{2}}-\frac{96}{r^{6 / 5}\left(\log \left(r^{2}\right)\right)^{3}}-\frac{12}{r\left(\log \left(r^{2}\right)\right)^{3}} \\
& +\frac{12}{r^{3 / 5} \log \left(r^{2}\right)}+\frac{288}{r^{3 / 5}\left(\log \left(r^{2}\right)\right)^{2}}+\frac{1212}{r^{3 / 5}\left(\log \left(r^{2}\right)\right)^{3}}+\frac{24}{r^{2 / 5}\left(\log \left(r^{2}\right)\right)^{2}} \\
& +\frac{384}{r^{2 / 5}\left(\log \left(r^{2}\right)\right)^{3}}+\frac{24}{r^{1 / 5}\left(\log \left(r^{2}\right)\right)^{3}}-4 \text {, } \\
& A_{20}^{*}(r) \approx \frac{4}{r^{9 / 5}\left(\log \left(r^{2}\right)\right)^{3}}-\frac{12}{r^{6 / 5}\left(\log \left(r^{2}\right)\right)^{2}}-\frac{96}{r^{6 / 5}\left(\log \left(r^{2}\right)\right)^{3}}-\frac{12}{r\left(\log \left(r^{2}\right)\right)^{3}} \\
& +\frac{12}{r^{3 / 5} \log \left(r^{2}\right)}+\frac{296}{r^{3 / 5}\left(\log \left(r^{2}\right)\right)^{2}}+\frac{1212}{r^{3 / 5}\left(\log \left(r^{2}\right)\right)^{3}}+\frac{28}{r^{2 / 5}\left(\log \left(r^{2}\right)\right)^{2}} \\
& +\frac{384}{r^{2 / 5}\left(\log \left(r^{2}\right)\right)^{3}}+\frac{24}{r^{1 / 5}\left(\log \left(r^{2}\right)\right)^{3}}-4 \\
& A_{19}^{*}(r) \approx-\frac{4}{r^{9 / 5}\left(\log \left(r^{2}\right)\right)^{3}}+\frac{8}{r^{6 / 5}\left(\log \left(r^{2}\right)\right)^{2}}+\frac{96}{r^{6 / 5}\left(\log \left(r^{2}\right)\right)^{3}}+\frac{12}{r\left(\log \left(r^{2}\right)\right)^{3}} \\
& -\frac{4}{r^{3 / 5} \log \left(r^{2}\right)}-\frac{224}{r^{3 / 5}\left(\log \left(r^{2}\right)\right)^{2}}-\frac{1212}{r^{3 / 5}\left(\log \left(r^{2}\right)\right)^{3}}-\frac{16}{r^{2 / 5}\left(\log \left(r^{2}\right)\right)^{2}} \\
& -\frac{384}{r^{2 / 5}\left(\log \left(r^{2}\right)\right)^{3}}-\frac{24}{r^{1 / 5}\left(\log \left(r^{2}\right)\right)^{3}} \text {, }
\end{aligned}
$$




$$
\begin{aligned}
A_{18}^{*}(r) \approx & \frac{4}{r^{9 / 5}\left(\log \left(r^{2}\right)\right)^{3}}-\frac{12}{r^{6 / 5}\left(\log \left(r^{2}\right)\right)^{2}}-\frac{96}{r^{6 / 5}\left(\log \left(r^{2}\right)\right)^{3}}-\frac{12}{r\left(\log \left(r^{2}\right)\right)^{3}} \\
& +\frac{12}{r^{3 / 5} \log \left(r^{2}\right)}+\frac{296}{r^{3 / 5}\left(\log \left(r^{2}\right)\right)^{2}}+\frac{1212}{r^{3 / 5}\left(\log \left(r^{2}\right)\right)^{3}}+\frac{28}{r^{2 / 5}\left(\log \left(r^{2}\right)\right)^{2}} \\
& +\frac{384}{r^{2 / 5}\left(\log \left(r^{2}\right)\right)^{3}}+\frac{24}{r^{1 / 5}\left(\log \left(r^{2}\right)\right)^{3}}-4,
\end{aligned}
$$

$$
\begin{aligned}
A_{15}^{*}(r) \approx & -\frac{4}{r^{9 / 5}\left(\log \left(r^{2}\right)\right)^{3}}+\frac{8}{r^{6 / 5}\left(\log \left(r^{2}\right)\right)^{2}}+\frac{96}{r^{6 / 5}\left(\log \left(r^{2}\right)\right)^{3}}+\frac{12}{r\left(\log \left(r^{2}\right)\right)^{3}} \\
& -\frac{4}{r^{3 / 5} \log \left(r^{2}\right)}-\frac{208}{r^{3 / 5}\left(\log \left(r^{2}\right)\right)^{2}}-\frac{1212}{r^{3 / 5}\left(\log \left(r^{2}\right)\right)^{3}}-\frac{20}{r^{2 / 5}\left(\log \left(r^{2}\right)\right)^{2}} \\
& -\frac{384}{r^{2 / 5}\left(\log \left(r^{2}\right)\right)^{3}}-\frac{24}{r^{1 / 5}\left(\log \left(r^{2}\right)\right)^{3}},
\end{aligned}
$$$$
\begin{aligned}
A_{14}^{*}(r) \approx & -\frac{4}{r^{9 / 5}\left(\log \left(r^{2}\right)\right)^{3}}+\frac{8}{r^{6 / 5}\left(\log \left(r^{2}\right)\right)^{2}}+\frac{96}{r^{6 / 5}\left(\log \left(r^{2}\right)\right)^{3}}+\frac{12}{r\left(\log \left(r^{2}\right)\right)^{3}} \\
& -\frac{4}{r^{3 / 5} \log \left(r^{2}\right)}-\frac{216}{r^{3 / 5}\left(\log \left(r^{2}\right)\right)^{2}}-\frac{1212}{r^{3 / 5}\left(\log \left(r^{2}\right)\right)^{3}}-\frac{24}{r^{2 / 5}\left(\log \left(r^{2}\right)\right)^{2}} \\
& -\frac{384}{r^{2 / 5}\left(\log \left(r^{2}\right)\right)^{3}}-\frac{24}{r^{1 / 5}\left(\log \left(r^{2}\right)\right)^{3}},
\end{aligned}
$$$$
A_{13}^{*}(r) \approx \frac{4}{r^{9 / 5}\left(\log \left(r^{2}\right)\right)^{3}}-\frac{4}{r^{6 / 5}\left(\log \left(r^{2}\right)\right)^{2}}-\frac{96}{r^{6 / 5}\left(\log \left(r^{2}\right)\right)^{3}}
$$$$
\begin{aligned}
& -\frac{12}{r\left(\log \left(r^{2}\right)\right)^{3}}+\frac{144}{r^{3 / 5}\left(\log \left(r^{2}\right)\right)^{2}}+\frac{1212}{r^{3 / 5}\left(\log \left(r^{2}\right)\right)^{3}}+\frac{12}{r^{2 / 5}\left(\log \left(r^{2}\right)\right)^{2}} \\
& +\frac{384}{r^{2 / 5}\left(\log \left(r^{2}\right)\right)^{3}}+\frac{24}{r^{1 / 5}\left(\log \left(r^{2}\right)\right)^{3}},
\end{aligned}
$$

$$
\begin{aligned}
A_{11}^{*}(r) \approx & \frac{4}{r^{9 / 5}\left(\log \left(r^{2}\right)\right)^{3}}-\frac{12}{r^{6 / 5}\left(\log \left(r^{2}\right)\right)^{2}}-\frac{96}{r^{6 / 5}\left(\log \left(r^{2}\right)\right)^{3}}-\frac{12}{r\left(\log \left(r^{2}\right)\right)^{3}} \\
& +\frac{12}{r^{3 / 5} \log \left(r^{2}\right)}+\frac{288}{r^{3 / 5}\left(\log \left(r^{2}\right)\right)^{2}}+\frac{1212}{r^{3 / 5}\left(\log \left(r^{2}\right)\right)^{3}}+\frac{24}{r^{2 / 5}\left(\log \left(r^{2}\right)\right)^{2}} \\
& +\frac{384}{r^{2 / 5}\left(\log \left(r^{2}\right)\right)^{3}}+\frac{24}{r^{1 / 5}\left(\log \left(r^{2}\right)\right)^{3}}-4,
\end{aligned}
$$

$$
\begin{aligned}
A_{10}^{*}(r) \approx & -\frac{4}{r^{9 / 5}\left(\log \left(r^{2}\right)\right)^{3}}+\frac{8}{r^{6 / 5}\left(\log \left(r^{2}\right)\right)^{2}}+\frac{96}{r^{6 / 5}\left(\log \left(r^{2}\right)\right)^{3}}+\frac{12}{r\left(\log \left(r^{2}\right)\right)^{3}} \\
& -\frac{4}{r^{3 / 5} \log \left(r^{2}\right)}-\frac{224}{r^{3 / 5}\left(\log \left(r^{2}\right)\right)^{2}}-\frac{1212}{r^{3 / 5}\left(\log \left(r^{2}\right)\right)^{3}}-\frac{16}{r^{2 / 5}\left(\log \left(r^{2}\right)\right)^{2}} \\
& -\frac{384}{r^{2 / 5}\left(\log \left(r^{2}\right)\right)^{3}}-\frac{24}{r^{1 / 5}\left(\log \left(r^{2}\right)\right)^{3}},
\end{aligned}
$$




$$
\begin{aligned}
& A_{5}^{*}(r) \approx-\frac{4}{r^{9 / 5}\left(\log \left(r^{2}\right)\right)^{3}}+\frac{8}{r^{6 / 5}\left(\log \left(r^{2}\right)\right)^{2}}+\frac{96}{r^{6 / 5}\left(\log \left(r^{2}\right)\right)^{3}}+\frac{12}{r\left(\log \left(r^{2}\right)\right)^{3}} \\
& -\frac{4}{r^{3 / 5} \log \left(r^{2}\right)}-\frac{200}{r^{3 / 5}\left(\log \left(r^{2}\right)\right)^{2}}-\frac{1212}{r^{3 / 5}\left(\log \left(r^{2}\right)\right)^{3}}-\frac{16}{r^{2 / 5}\left(\log \left(r^{2}\right)\right)^{2}} \\
& -\frac{384}{r^{2 / 5}\left(\log \left(r^{2}\right)\right)^{3}}-\frac{24}{r^{1 / 5}\left(\log \left(r^{2}\right)\right)^{3}}, \\
& A_{4}^{*}(r) \approx-\frac{4}{r^{9 / 5}\left(\log \left(r^{2}\right)\right)^{3}}+\frac{8}{r^{6 / 5}\left(\log \left(r^{2}\right)\right)^{2}}+\frac{96}{r^{6 / 5}\left(\log \left(r^{2}\right)\right)^{3}}+\frac{12}{r\left(\log \left(r^{2}\right)\right)^{3}} \\
& -\frac{4}{r^{3 / 5} \log \left(r^{2}\right)}-\frac{208}{r^{3 / 5}\left(\log \left(r^{2}\right)\right)^{2}}-\frac{1212}{r^{3 / 5}\left(\log \left(r^{2}\right)\right)^{3}}-\frac{20}{r^{2 / 5}\left(\log \left(r^{2}\right)\right)^{2}} \\
& -\frac{384}{r^{2 / 5}\left(\log \left(r^{2}\right)\right)^{3}}-\frac{24}{r^{1 / 5}\left(\log \left(r^{2}\right)\right)^{3}}, \\
& A_{3}^{*}(r) \approx \frac{8}{r^{9 / 5}\left(\log \left(r^{2}\right)\right)^{3}}-\frac{8}{r^{6 / 5}\left(\log \left(r^{2}\right)\right)^{2}}-\frac{192}{r^{6 / 5}\left(\log \left(r^{2}\right)\right)^{3}} \\
& -\frac{24}{r\left(\log \left(r^{2}\right)\right)^{3}}+\frac{272}{r^{3 / 5}\left(\log \left(r^{2}\right)\right)^{2}}+\frac{2424}{r^{3 / 5}\left(\log \left(r^{2}\right)\right)^{3}}+\frac{16}{r^{2 / 5}\left(\log \left(r^{2}\right)\right)^{2}} \\
& +\frac{768}{r^{2 / 5}\left(\log \left(r^{2}\right)\right)^{3}}+\frac{48}{r^{1 / 5}\left(\log \left(r^{2}\right)\right)^{3}}, \\
& A_{2}^{*}(r) \approx \frac{4}{r^{9 / 5}\left(\log \left(r^{2}\right)\right)^{3}}-\frac{4}{r^{6 / 5}\left(\log \left(r^{2}\right)\right)^{2}}-\frac{96}{r^{6 / 5}\left(\log \left(r^{2}\right)\right)^{3}} \\
& -\frac{12}{r\left(\log \left(r^{2}\right)\right)^{3}}+\frac{144}{r^{3 / 5}\left(\log \left(r^{2}\right)\right)^{2}}+\frac{1212}{r^{3 / 5}\left(\log \left(r^{2}\right)\right)^{3}}+\frac{12}{r^{2 / 5}\left(\log \left(r^{2}\right)\right)^{2}} \\
& +\frac{384}{r^{2 / 5}\left(\log \left(r^{2}\right)\right)^{3}}+\frac{24}{r^{1 / 5}\left(\log \left(r^{2}\right)\right)^{3}}, \\
& A_{1}^{*}(r) \approx-\frac{4}{r^{9 / 5}\left(\log \left(r^{2}\right)\right)^{3}}+\frac{96}{r^{6 / 5}\left(\log \left(r^{2}\right)\right)^{3}} \\
& +\frac{12}{r\left(\log \left(r^{2}\right)\right)^{3}}-\frac{72}{r^{3 / 5}\left(\log \left(r^{2}\right)\right)^{2}}-\frac{1212}{r^{3 / 5}\left(\log \left(r^{2}\right)\right)^{3}} \\
& -\frac{384}{r^{2 / 5}\left(\log \left(r^{2}\right)\right)^{3}}-\frac{24}{r^{1 / 5}\left(\log \left(r^{2}\right)\right)^{3}} \text {. }
\end{aligned}
$$

Again

$$
\begin{aligned}
A_{j}^{*}(r) \approx & \frac{a_{j}}{r^{9 / 5}\left(\log \left(r^{2}\right)\right)^{3}}+\frac{b_{j}}{r^{6 / 5}\left(\log \left(r^{2}\right)\right)^{2}}+\frac{c_{j}}{r^{6 / 5}\left(\log \left(r^{2}\right)\right)^{3}}+\frac{d_{j}}{r\left(\log \left(r^{2}\right)\right)^{3}} \\
& \frac{e_{j}}{r^{3 / 5} \log \left(r^{2}\right)}+\frac{f_{j}}{r^{3 / 5}\left(\log \left(r^{2}\right)\right)^{2}}+\frac{g_{j}}{r^{3 / 5}\left(\log \left(r^{2}\right)\right)^{3}}+\frac{h_{j}}{r^{2 / 5}\left(\log \left(r^{2}\right)\right)^{2}} \\
& +\frac{i_{j}}{r^{2 / 5}\left(\log \left(r^{2}\right)\right)^{3}}+\frac{k_{j}}{r^{1 / 5}\left(\log \left(r^{2}\right)\right)^{3}}+l_{j}
\end{aligned}
$$

means that 


$$
\begin{gathered}
\lim _{r \rightarrow 0^{+}} A_{j}^{*}(r) r^{9 / 5}\left(\log \left(r^{2}\right)\right)^{3}=a_{j}, \\
\lim _{r \rightarrow 0^{+}}\left(A_{j}^{*}(r)-\frac{a_{j}}{r^{9 / 5}\left(\log \left(r^{2}\right)\right)^{3}}\right) r^{6 / 5}\left(\log \left(r^{2}\right)\right)^{2}=b_{j}, \\
\lim _{r \rightarrow 0^{+}}\left(A_{j}^{*}(r)-\frac{a_{j}}{r^{9 / 5}\left(\log \left(r^{2}\right)\right)^{3}}-\frac{b_{j}}{r^{6 / 5}\left(\log \left(r^{2}\right)\right)^{2}}-\frac{c_{j}}{r^{6 / 5}\left(\log \left(r^{2}\right)\right)^{3}}\right. \\
-\frac{d_{j}}{r\left(\log \left(r^{2}\right)\right)^{3}}-\frac{e_{j}}{r^{3 / 5} \log \left(r^{2}\right)}-\frac{f_{j}}{r^{3 / 5}\left(\log \left(r^{2}\right)\right)^{2}}-\frac{g_{j}}{r^{3 / 5}\left(\log \left(r^{2}\right)\right)^{3}} \\
\left.-\frac{h_{j}}{r^{2 / 5}\left(\log \left(r^{2}\right)\right)^{2}}-\frac{i_{j}}{r^{2 / 5}\left(\log \left(r^{2}\right)\right)^{3}}-\frac{k_{j}}{r^{1 / 5}\left(\log \left(r^{2}\right)\right)^{3}}\right)=l_{j}
\end{gathered}
$$

(to simplify the calculations one can put $r=q^{10}$ and then find $\lim _{q \rightarrow 0} \ldots$ ).

The expansions of the other terms are:

$$
\begin{aligned}
& A_{24}^{*}(r) \approx-\frac{4}{r^{6 / 5}\left(\log \left(r^{2}\right)\right)^{2}}+\frac{12}{r^{3 / 5} \log \left(r^{2}\right)}+\frac{64}{r^{3 / 5}\left(\log \left(r^{2}\right)\right)^{2}}+\frac{8}{r^{2 / 5}\left(\log \left(r^{2}\right)\right)^{2}}-12, \\
& A_{22}^{*}(r) \approx \frac{8}{r^{6 / 5}\left(\log \left(r^{2}\right)\right)^{2}}-\frac{8}{r^{3 / 5} \log \left(r^{2}\right)}-\frac{64}{r^{3 / 5}\left(\log \left(r^{2}\right)\right)^{2}}-\frac{8}{r^{2 / 5}\left(\log \left(r^{2}\right)\right)^{2}}+4 \\
& A_{17}^{*}(r) \approx \frac{4}{r^{6 / 5}\left(\log \left(r^{2}\right)\right)^{2}}-\frac{8}{r^{3 / 5} \log \left(r^{2}\right)}-\frac{64}{r^{3 / 5}\left(\log \left(r^{2}\right)\right)^{2}}-\frac{8}{r^{2 / 5}\left(\log \left(r^{2}\right)\right)^{2}}+4 \\
& A_{16}^{*}(r) \approx-\frac{4}{r^{6 / 5}\left(\log \left(r^{2}\right)\right)^{2}}+\frac{4}{r^{3 / 5} \log \left(r^{2}\right)}+\frac{64}{r^{3 / 5}\left(\log \left(r^{2}\right)\right)^{2}}+\frac{8}{r^{2 / 5}\left(\log \left(r^{2}\right)\right)^{2}} \\
& A_{12}^{*}(r) \approx \frac{4}{r^{6 / 5}\left(\log \left(r^{2}\right)\right)^{2}}-\frac{8}{r^{3 / 5} \log \left(r^{2}\right)}-\frac{64}{r^{3 / 5}\left(\log \left(r^{2}\right)\right)^{2}}-\frac{8}{r^{2 / 5}\left(\log \left(r^{2}\right)\right)^{2}}+4 \\
& A_{9}^{*}(r) \approx \frac{2}{r^{6 / 5}\left(\log \left(r^{2}\right)\right)^{2}}-\frac{4}{r^{3 / 5} \log \left(r^{2}\right)}-\frac{32}{r^{3 / 5}\left(\log \left(r^{2}\right)\right)^{2}}-\frac{4}{r^{2 / 5}\left(\log \left(r^{2}\right)\right)^{2}}+2 \\
& A_{8}^{*}(r) \approx-\frac{8}{r^{6 / 5}\left(\log \left(r^{2}\right)\right)^{2}}+\frac{8}{r^{3 / 5} \log \left(r^{2}\right)}+\frac{128}{r^{3 / 5}\left(\log \left(r^{2}\right)\right)^{2}}+\frac{16}{r^{2 / 5}\left(\log \left(r^{2}\right)\right)^{2}} \\
& A_{7}^{*}(r) \approx-\frac{4}{r^{6 / 5}\left(\log \left(r^{2}\right)\right)^{2}}+\frac{4}{r^{3 / 5} \log \left(r^{2}\right)}+\frac{64}{r^{3 / 5}\left(\log \left(r^{2}\right)\right)^{2}}+\frac{8}{r^{2 / 5}\left(\log \left(r^{2}\right)\right)^{2}} \\
& A_{6}^{*}(r) \approx \frac{6}{r^{6 / 5}\left(\log \left(r^{2}\right)\right)^{2}}-\frac{96}{r^{3 / 5}\left(\log \left(r^{2}\right)\right)^{2}}-\frac{12}{r^{2 / 5}\left(\log \left(r^{2}\right)\right)^{2}}
\end{aligned}
$$

Finally one obtains

$$
\sum_{j=1}^{24} A_{j}^{*}(r) \approx 2 .
$$

Therefore

$$
\lim _{r \rightarrow 0^{+}} A_{j}(r)=2 .
$$


Observation 1. One has

$$
\begin{aligned}
R_{P_{r}}\left(e^{i \theta} z\right) & =R_{P_{r}}(z) \quad \text { for all } \theta \in(0,2 \pi], \\
R_{P_{r}}(z) & =R_{P_{r}}(r / z) .
\end{aligned}
$$

One easily checks that both $z \mapsto e^{i \theta} z$ and $z \mapsto r / z$ are holomorphic automorphisms of $P_{r}$. Now everything follows from the invariance property of the holomorphic sectional curvature of the Bergman metric. One moreover sees that for the choice $z=r^{7 / 10}=\frac{r}{r^{3 / 10}}$ the equality

$$
\lim _{r \rightarrow 0^{+}} R_{P_{r}}\left(r^{7 / 10}\right)=2
$$

is also true, which is also revealed by the figures.

Observation 2. One has

$$
R_{\left\{\rho_{1}<\left|w-z_{0}\right|<\rho_{2}\right\}}(z)=R_{P_{\rho_{1} / \rho_{2}}}\left(\frac{z-z_{0}}{\rho_{2}}\right) .
$$

This is also a simple consequence of the fact that $z \mapsto\left(z-z_{0}\right) / \rho_{2}$ is biholomorphic between the two domains.

2. An example. Let $\left\{R_{j}\right\}_{j=1}^{\infty},\left\{r_{j}\right\}_{j=1}^{\infty},\left\{s_{j}\right\}_{j=1}^{\infty}$ be three sequences of positive real numbers that obey the following conditions:

$$
\sum_{j=1}^{\infty} R_{j}<\infty
$$

(ii) $\quad r_{1}<R_{1} / 2, \quad r_{j} / R_{j}$ is decreasing and $\lim _{j \rightarrow \infty} r_{j} / R_{j}=0$,

(iii) $s_{j}<\min \left\{2 R_{j} \sin (0.07 \pi), 2 R_{j+1} \sin (0.07 \pi)\right.$,

$$
\left.R_{j}-\left(r_{j} / R_{j}\right)^{3 / 10}, R_{j+1}-\left(r_{j+1} / R_{j+1}\right)^{3 / 10}\right\} .
$$

Consider the domain $\Omega=\bigcup_{j=1}^{\infty} \Omega_{j}$, where $\Omega_{1}=\left\{z: r_{1}<|z|<R_{1}\right\}$, and each $\Omega_{j}$ is an annulus with inner radius $r_{j}$ and outer radius $R_{j}$ which is centred on the positive real axis, to the right of $\Omega_{j-1}$ and overlaps with $\Omega_{j-1}$ in such a way that the segment joining the two intersection points of the circles with radii $R_{j}$ and $R_{j-1}$ has length $s_{j-1}$. There are now two possible choices for $\Omega_{j}$ : one for which most of $\Omega_{j}$ lies inside $\Omega_{j-1}$, and one when most of it lies outside. We consider the latter one. The choice of $s_{j}$ ensures that the discs with radii $r_{j}$ and $r_{j-1}$ do not overlap.

By (i), $\Omega$ is bounded.

Let $\Omega_{j}^{\prime}=\Omega_{j} \backslash\left(K_{1} \cup K_{2}\right)$, where $K_{1}$ is a disc of radius $s_{j-1} / 2$, centred at the midpoint of the segment joining the intersection points of the circles with radii $R_{j}$ and $R_{j-1}$ (the outer boundaries of the annuli $\Omega_{j}$ and $\Omega_{j-1}$ ), while $K_{2}$ has radius $s_{j} / 2$ and is centred at the midpoint of the segment joining the intersection points of the circles with radii $R_{j}$ and $R_{j+1}$. 


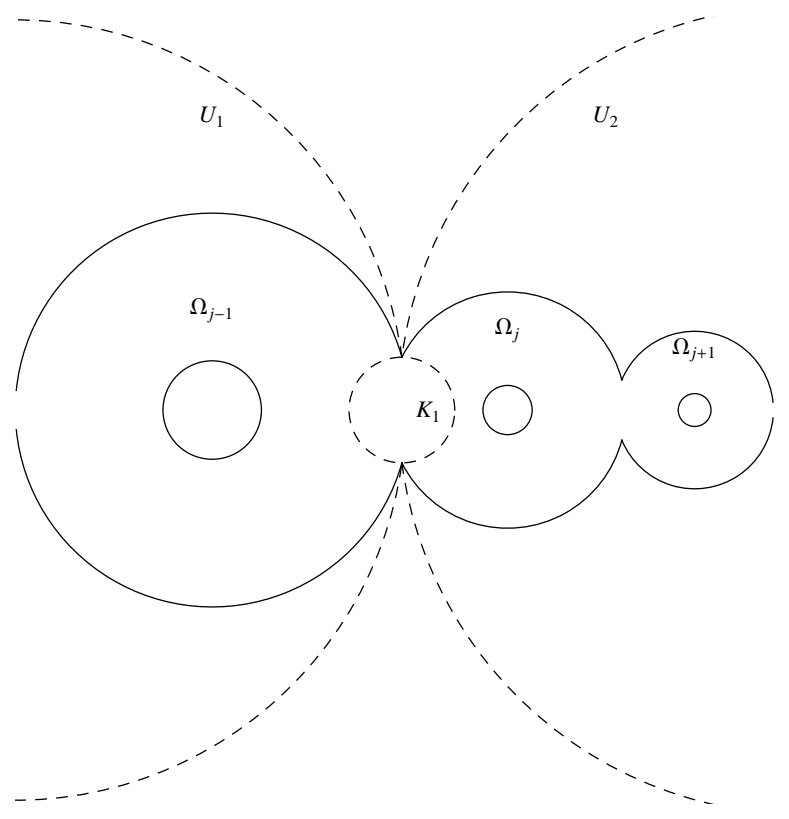

Fig. 5. Part of the domain $\Omega$

We begin with a lemma:

Lemma 2.1. Let $z_{0} \in \Omega_{j}^{\prime}$. Then the sublevel set of the Green function $G_{\Omega}\left(z, z_{0}\right)$,

$$
\left\{G_{\Omega}\left(z, z_{0}\right)<-1\right\}
$$

is entirely contained in $\Omega_{j}\left({ }^{2}\right)$.

Proof. The Green function is obviously decreasing with respect to domain inclusions. Therefore it is enough to show that $\left\{G_{U}\left(z, z_{0}\right)<-1\right\} \subset \Omega_{j}$ for some $U \supset \Omega$.

For simplicity one can translate $\Omega$ so that the upper intersection point of the circles with radii $R_{j-1}$ and $R_{j}$ is 0 . Choose two discs $U_{1}$ and $U_{2}$, with radii $\rho_{1}$ and $\rho_{2}$, whose boundaries intersect at 0 and $-i s_{j-1}$ such that $\Omega \subset U_{1} \cup U_{2}$. Clearly $\rho_{1} \geq R_{j-1}$ and $\rho_{2}>R_{j}$. The function $G_{U_{1} \cup U_{2}}\left(z, z_{0}\right)$ can be explicitly calculated as $h \circ f$, where

$$
h(w):=\log \left|\frac{w-f\left(z_{0}\right)}{1-w \overline{f\left(z_{0}\right)}}\right|
$$

$\left({ }^{2}\right)$ Informally: the sublevel set cannot "escape" through the very narrow passage between the annuli. 
and

$$
f(z):=\frac{\left(\left(\frac{1}{z}-\frac{i}{s_{j-1}}\right) e^{-i \frac{\pi}{2}} e^{i \frac{\beta-\alpha}{2}}\right)^{\frac{\pi}{2 \pi-\alpha-\beta}}-1}{\left(\left(\frac{1}{z}-\frac{i}{s_{j-1}}\right) e^{-i \frac{\pi}{2}} e^{i \frac{\beta-\alpha}{2}}\right)^{\frac{\pi}{2 \pi-\alpha-\beta}}+1}
$$

is the mapping that transforms $U_{1} \cup U_{2}$ conformally into the unit disc. Here $\alpha=\arcsin \frac{s_{j-1}}{2 \rho_{2}}, \beta=\arcsin \frac{s_{j-1}}{2 \rho_{1}}$.

The image of $U_{2}$ under $f$ is the intersection of the unit disc with a disc centred at the negative imaginary axis, passing through $\{1\}$ and $\{-1\}$ and such that the angle between its tangent line at $\{-1\}$ and the line $-1+i t$ is exactly $\frac{\pi}{2 \pi-\beta-\alpha} \frac{\beta+\alpha}{2}$. The image of $U_{1}$ is exactly the conjugate of this set. Now

$$
f\left(\left\{G_{U_{1} \cup U_{2}}\left(z, z_{0}\right)<-1\right\}\right)=\left\{w:\left|\frac{w-f\left(z_{0}\right)}{1-w \overline{f\left(z_{0}\right)}}\right|<e^{-1}\right\},
$$

which is the disc

$$
\left|w-f\left(z_{0}\right) \frac{1-e^{-2}}{1-e^{-2}\left|f\left(z_{0}\right)\right|^{2}}\right|<\frac{e^{-1}\left(1-\left|f\left(z_{0}\right)\right|^{2}\right)}{1-e^{-2}\left|f\left(z_{0}\right)\right|^{2}} .
$$

Now if

$$
z_{0} \in U_{2}, \quad\left|\operatorname{Im} f\left(z_{0}\right)\right| \frac{1-e^{-2}}{1-e^{-2}\left|f\left(z_{0}\right)\right|^{2}} \geq \frac{e^{-1}\left(1-\left|f\left(z_{0}\right)\right|^{2}\right)}{1-e^{-2}\left|f\left(z_{0}\right)\right|^{2}}
$$

then this disc will stay in the lower halfdisc (and hence in $f\left(U_{2}\right)$ ).

This inequality transforms easily into

$$
\left|f\left(z_{0}\right)-i \frac{1-e^{-2}}{2 e^{-1}}\right| \geq \frac{1+e^{-2}}{2 e^{-1}}, \quad \operatorname{Im} f\left(z_{0}\right)<0 .
$$

So this is the set enclosed by the arcs of the unit circle and a circle that passes through $\{-1\}$ and $\{1\}$, and the angle between the real axis and the tangent line at $\{-1\}$ is (in absolute value) $\arccos \frac{1-e^{-2}}{1+e^{-2}} \approx 0.22 \pi<0.23 \pi$.

On the other hand the image of the disc $\left|z+i \frac{s_{j-1}}{2}\right|<\frac{s_{j-1}}{2}\left(K_{1}\right)$ is the set enclosed by two circular arcs joining $\{-1\}$ with $\{1\}$ characterized by the angle between the real axis and the tangent line at $\{-1\}\left(\frac{\pi-\beta+\alpha}{2} \frac{\pi}{2 \pi-\beta-\alpha}\right.$ and $(-) \frac{\pi+\beta-\alpha}{2} \frac{\pi}{2 \pi-\beta-\alpha}$ respectively) and we see that if both $\alpha$ and $\beta$ are smaller than $0.07 \pi$ then the slope of the lower arc is greater than $0.23 \pi$ and hence lies in the region defined by (2.1). Since $z_{0} \notin K_{1}, f\left(z_{0}\right)$ must lie below the arc in question and hence in the desired region.

It remains to observe that the condition $\alpha, \beta<0.07 \pi$ is fulfilled by the choice of $s_{j-1}$ and that one can carry out the same argument for $K_{2}$.

Let $\zeta$ be the rightmost boundary point of $\Omega$ (the accumulation point of the annuli). Now one can give the proof of the main theorem.

Proof. It is clear that $J_{i, \Omega}(z) \leq J_{i, \Omega_{j}}(z)$ for all $z \in \Omega_{j}, i=0,1,2$ and for all $j$ (see $0.3-0.5)$. 
Let $z_{0} \in \Omega_{j}^{\prime}$ and let $f_{i}(z)$ be the corresponding function that realizes the supremum in the definition of $J_{i, \Omega_{j}}$. We have

$$
f_{i}(z) \in \mathcal{O}\left(\Omega_{j}\right) \cap L^{2}\left(\Omega_{j}\right), \quad \int_{\Omega_{j}}\left|f_{i}\right|^{2} \leq 1, \quad f_{i}^{(k)}\left(z_{0}\right)=0, \quad k=0, \ldots, i-1 .
$$

Let $\chi$ be a real smooth function of a real variable such that $\chi(x)=0$ for $x>-1, \chi(x)=1$ for $x<-2,0 \leq \chi(x) \leq 1$ for $-2 \leq x \leq-1$, and $\left|\chi^{\prime}(x)\right|<C$ globally for some positive constant $C$.

By Lemma 2.1 the $(0,1)$-form $\bar{\partial}\left(\chi \circ G_{\Omega}\left(z, z_{0}\right)\right) f_{i}(z)$ can be extended (trivially) to a smooth ( $\bar{\partial}$-closed) form on the whole $\Omega$.

Note that $e^{G_{\Omega}\left(z, z_{0}\right)}$ is a subharmonic function that satisfies

$$
\frac{\partial^{2}}{\partial z \partial \bar{z}} e^{G_{\Omega}\left(z, z_{0}\right)} \geq\left|\frac{\partial}{\partial z} e^{G_{\Omega}\left(z, z_{0}\right)}\right|^{2}
$$

in the weak sense. Therefore by the Donnelly-Fefferman estimate (see [1] and especially [2], where the passing from smooth to nonsmooth data is presented very clearly) one can find a solution $v$ of the $\bar{\partial}$ problem

$$
\bar{\partial} v_{i}=\bar{\partial}\left(\chi \circ G_{\Omega}\left(z, z_{0}\right)\right) f_{i}(z)
$$

in $\Omega$, with

$$
\begin{aligned}
\int_{\Omega}\left|v_{i}\right|^{2} e^{-2(i+1) G_{\Omega}\left(z, z_{0}\right)} & \leq C^{\prime} \int_{\Omega} \frac{\left|\bar{\partial}\left(\chi \circ G_{\Omega}\left(z, z_{0}\right)\right) f_{i}(z)\right|^{2}}{\frac{\partial^{2}}{\partial z \partial \bar{z}} e^{G_{\Omega}\left(z, z_{0}\right)}} e^{-2(i+1) G_{\Omega}\left(z, z_{0}\right)} \\
& \leq C^{\prime} \int_{\left\{-2<G_{\Omega}\left(z, z_{0}\right)<-1\right\}} \frac{C^{2}\left|f_{i}\right|^{2}}{e^{(2 i+3) G_{\Omega}\left(z, z_{0}\right)}} \leq C^{\prime} C^{2} e^{2(2 i+3)},
\end{aligned}
$$

where $C^{\prime}$ is a universal constant.

Moreover $v_{i}$ is holomorphic in a neighbourhood of $z_{0}$, and the above inequality ensures that $v_{i}^{(k)}\left(z_{0}\right)=0, k=0, \ldots, i$.

The function $g_{i}=\left(\chi \circ G_{\Omega}\left(z, z_{0}\right)\right) f_{i}(z)-v_{i}$ is holomorphic in $\Omega$, agrees to the $i$ th order with $f_{i}$ at $z_{0}$ and

$$
\begin{aligned}
\left(\int_{\Omega}\left|g_{i}\right|^{2}\right)^{1 / 2} & \leq\left(\int_{\Omega}\left|v_{i}\right|^{2}\right)^{1 / 2}+\left(\int_{\Omega}\left|\left(\chi \circ G_{\Omega}\left(z, z_{0}\right)\right) f_{i}(z)\right|^{2}\right)^{1 / 2} \\
& \leq\left(\int_{\Omega}\left|v_{i}\right|^{2} e^{-2(i+1) G_{\Omega}\left(z, z_{0}\right)}\right)^{1 / 2}+1 \leq 1+\sqrt{C^{\prime} C^{2} e^{2(2 i+3)}} .
\end{aligned}
$$

The choice of the function $\frac{g_{i}(z)}{1+\sqrt{C^{\prime} C^{2} e^{2(2 i+3)}}}$ shows that

$$
J_{i, \Omega}(z) \geq \frac{J_{i, \Omega_{j}}(z)}{1+\sqrt{C^{\prime} C^{2} e^{2(2 i+3)}}}
$$

for all $z \in \Omega_{j}^{\prime}$. We note that this gives us a lower bound for the expression $J_{i, \Omega}(z) / J_{i, \Omega_{j}}(z)$ which is independent of $j$. 
Hence for every $z \in \Omega$ that lies in $\Omega_{j}^{\prime}$ for some $j$,

$$
\begin{aligned}
2-R_{\Omega}(z) & =\frac{J_{0, \Omega}(z) J_{2, \Omega}(z)}{J_{1, \Omega}(z)^{2}} \leq \frac{\left(1+\sqrt{C^{\prime} C^{2} e^{10}}\right)^{2} J_{0, \Omega_{j}}(z) J_{2, \Omega_{j}}(z)}{J_{1, \Omega_{j}}(z)^{2}} \\
& =C^{\prime \prime}\left(2-R_{\Omega_{j}}(z)\right)
\end{aligned}
$$

and

$$
2-R_{\Omega}(z) \geq \frac{J_{0, \Omega_{j}}(z) J_{2, \Omega_{j}}(z)}{J_{1, \Omega_{j}}(z)^{2}\left(1+\sqrt{C^{\prime} C^{2} e^{14}}\right)\left(1+\sqrt{C^{\prime} C^{2} e^{6}}\right)}=C^{\prime \prime \prime}\left(2-R_{\Omega_{j}}(z)\right) .
$$

Let $z_{j}^{\prime}$ be the point in $\Omega_{j}$ (and in $\Omega_{j}^{\prime}$, by (iii)) that corresponds to the point $R_{j} \sqrt{r_{j} / R_{j}}+0 i$ in the annulus $\left\{r_{j}<|z|<R_{j}\right\}$. By analogy we define $z_{j}^{\prime \prime}$ to be the point that corresponds to $R_{j}\left(r_{j} / R_{J}\right)^{3 / 10}$. Then

$$
\begin{aligned}
& \limsup _{\Omega \ni z \rightarrow \zeta} R_{\Omega}(z)=\limsup _{j \rightarrow \infty} R_{\Omega}\left(z_{j}^{\prime \prime}\right)=2, \\
& \liminf _{\Omega \ni z \rightarrow \zeta} R_{\Omega}(z)=\liminf _{j \rightarrow \infty} R_{\Omega}\left(z_{j}^{\prime}\right)=-\infty,
\end{aligned}
$$

by Theorem 0.1 and Observation 2 .

OBSERVATION 3. $\Omega$ defined as above is hyperconvex.

Hyperconvexity is the same as regularity (with respect to the Dirichlet problem) in dimension 1 . One easily constructs barrier functions at each boundary point of $\Omega$ and by Perron's method $\Omega$ is regular.

This is quite unexpected since it is known that both the Bergman kernel in arbitrary dimension (see [13]) and the Bergman metric in dimension 1 behave in a quite predictable way in hyperconvex domains (see [14]; in higher dimensions it is rather B-regularity than hyperconvexity that one has to have in mind, but the problems are not completely settled; see [3], [7], [6]).

Acknowledgments. I would like to thank Professor Zbigniew Błocki for numerous suggestions and encouragement. I would also like to thank Professor Włodzimierz Zwonek for helpful conversations on the topic and for finding a serious mistake in a previous version of the paper.

The author was supported by the Polish Ministry of Science and Higher Education Grant N N201 271235.

\section{References}

[1] B. Berndtsson, The extension theorem of Ohsawa-Takegoshi and the theorem of Donnelly-Fefferman, Ann. Inst. Fourier (Grenoble) 46 (1996), 1083-1094.

[2] Z. Błocki, The Bergman metric and the pluricomplex Green function, Trans. Amer. Math. Soc. 357 (2005), 2613-2625.

[3] Z. Błocki and P. Pflug, Hyperconvexity and Bergman completeness, Nagoya Math. J. 151 (1998), 221-225. 
[4] D. W. Catlin, Estimates of invariant metrics on pseudoconvex domains of dimension two, Math. Z. 200 (1989), 429-466.

[5] B. Y. Chen and H. J. Lee, Bergman kernel and complex singularity exponent, Sci. China Ser. A 52 (2009), 2590-2603.

[6] K. Diederich and G. Herbort, Quantitative estimates for the Green function and an application to the Bergman metric, Ann. Inst. Fourier (Grenoble) 50 (2000), 1205-1228.

[7] G. Herbort, The Bergman metric on hyperconvex domains, Math. Z. 232 (1999), 183-196.

[8] - Localization lemmas for the Bergman metric at plurisubharmonic peak points, Nagoya Math. J. 171 (2003), 107-125.

[9] -, An example of a pseudoconvex domain whose holomorphic sectional curvature of the Bergman metric is unbounded, Ann. Polon. Math. 92 (2007), 29-39.

[10] M. Jarnicki and P. Pflug, Invariant Distances and Metrics in Complex Analysis, de Gruyter Exp. Math. 9, de Gruyter, Berlin, 1993.

[11] P. F. Klembeck, Kähler metrics of negative curvature, the Bergmann metric near the boundary, and the Kobayashi metric on smooth bounded strictly pseudoconvex sets, Indiana Univ. Math. J. 27 (1978), 275-282.

[12] B. Ya. Lebed', Estimates of the curvature of a Bergman metric that is invariant under biholomorphic mappings, Funktsional. Anal. i Prilozhen. 5 (1971), no. 3, 100101 (in Russian).

[13] T. Ohsawa, On the Bergman kernel of hyperconvex domains, Nagoya Math. J. 129 (1993), 43-52.

[14] P. Pflug and W. Zwonek, Logarithmic capacity and Bergman functions, Arch. Math. (Basel) 80 (2003), 536-552.

[15] W. Zwonek, Asymptotic behavior of the sectional curvature of the Bergman metric for annuli, Ann. Polon. Math., to appear.

Żywomir Dinew

Institute of Mathematics

Jagiellonian University

Łojasiewicza 6

30-348 Kraków, Poland

E-mail: Zywomir.Dinew@im.uj.edu.pl

Received 24.6.2009

and in final form 19.3.2010 
